\title{
EXPOSÉ SUR LES NIVEAUX EXCITÉS 0+
}

\author{
Par - J. YOCCOZ, \\ Faculté des Sciences de Strasbourg.
}

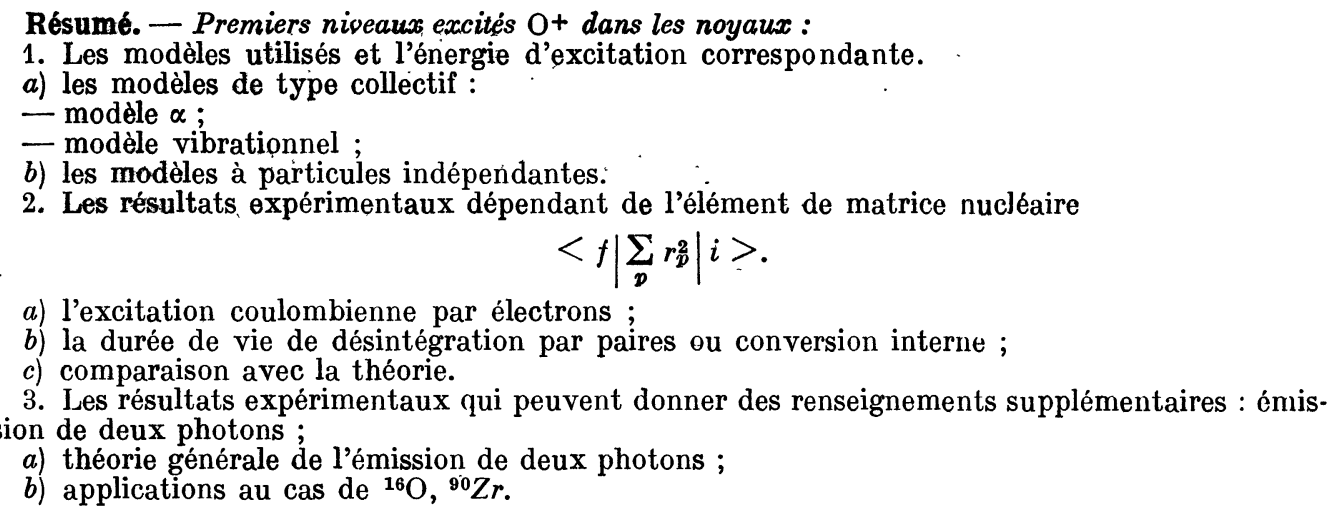

Abstract. - First $\mathrm{O}+$ excited levels in nuclei.

1. Models used and corresponding excitation.

a) collective type model :

- $\alpha$ model

- vibrationnal model ;

b) independent particles model.

2. Experimental results depending on the nuclear matrice element

$$
<f\left|\sum_{p} r_{p}^{2}\right| i>\text {. }
$$

a) Coulomb excitation by electrons.

b) Half-life of disintegration by pairs or internal conversion.

c) Comparison with theory.

3. Experimental results suitable for giving further information : two-photon emission ;

-a) general theory of emission of two-photons ;

b) application to ${ }^{16} \mathrm{O},{ }^{90} \mathrm{Zr}$.

Dans cet exposé, il ne sera question que des niveaux excités $\Theta^{+}$situés immédiatement au-dessus du niveau fondamental de même spin et parité, comme dans les noyaux doublement magiques $\left({ }^{16} \mathrm{O},{ }^{40} \mathrm{Ca}\right)$ ou simplement magiques comme ${ }^{90} \mathrm{Zr}$. Leur intérêt réside justement dans les questions qu'ils posent à propos de la dynamique des couches fermées du modèle à particules indépendantes. Les interprétations théoriques de ces niveaux peuvent être rangées grossièrement sous les deux rubriques classiques : mouvements collectifs ou excitations d'un nombre limité de particules. Dans le modèle $\alpha$, le plus ancien, le niveau $\mathrm{O}^{+}$de ${ }^{16} \mathrm{O}$ (et $\operatorname{de}{ }^{12} \mathrm{C}$ ) est interprété comme une dilatation de la structure $\alpha$, l'énergie d'excitation $\Delta$ fixant la fréquence d'oscillation, un test du modèle étant le fait de retrouver d'autres niveaux $\mathrm{O}^{+}$avec des énergies d'excitation - $2 \Delta, 3 \Delta$ etc. Malgré quelques succès $d u$ modèle élémentaire dans la prédiction des spins et parités des autres niveaux de ${ }^{16} \mathrm{O}$ 
(Dennisson 1954), ses bases théoriques sont trop faibles pour qu'il puisse être accepté. La même idée $d^{2}$ oscillation de dilatătion a été reprise sous d'autres formes, inspirées du traitement des autres types de mouvement collectif. Mais que l'on utilise une image hydrodynamique, que l'on traite le rayon du noyau comme un paramètre sur lequel on greffe un calcul variationnel (Griffin [5]) ou que l'on utilise n'importe quel autre artifice (Touchard [11]) le résultat est sensiblement toujours le même: cette oscillation monopolaire correspond à une énergie de $20 \mathrm{MeV}$ environ pour ${ }^{16} \mathrm{O}$, c'est-à-dire beaucoup plus grande que l'énergie du niveau qui nous intéresse, à savoir $6,06 \mathrm{MeV}$. Dans le cadre du modèle à particules indépendantes, des calculs assez complets ont été effectués par Bayman, Reiner et Sheline [1] et Talmi et Unna (1960) [10] en ce qui concerne ${ }^{90} \mathrm{Zr}$, les éléments de matrices de l'halmiltonien correspondant aux configurations $\left(2 p \frac{1}{2}\right)^{2} ; 2 p \frac{1}{2}, 1 g \frac{9}{2} ;\left(1 g \frac{9}{2}\right)^{2}$ étant évalués par comparaison avec les états excités des niveaux voisins. Le niveau $\mathrm{O}^{+}$de $1,75 \mathrm{MeV}$ s'intègre alors d'une façon satisfaisante dans l'ensemble du spectre. Les calculs en couplage intermédiaire de Kurath (1956) [7] ne donnent pas de résultats corrects pour le niveau $7,6 \mathrm{MeV}$ de ${ }^{12} \mathrm{C}$. Le cas de ${ }^{16} \mathrm{O}$ (celui de ${ }^{40} \mathrm{Ca}$ est analogue) a été fort discuté, mais aucun calcul réellement complet n'a été publié. En se bornant aux types d'excitation les plus économiques, on peut distinguer deux genres d'excitation : les excitations d'une seule particule $(1 p \rightarrow 2 p ; 1 s \rightarrow 2 s)$ et les excitations d'une paire de particules $\left((1 p)^{2} \rightarrow(2 s)^{2} ;(1 d)^{2}\right)$. Il est d'ailleurs parfaitement possible, en pondérant convenablement ces diverses configurations, de simuler les mouvements collectifs dont nous avons parlé plus haut. (Perring et skyrme (1956) [8] ; Ferrel et Visscher (1956) [3]).

Outre l'énergie d'excitation, la seule information expérimentale dont on disposait était l'élément de matrice dit monopolaire $<\psi_{f}\left|\sum_{1}^{z} r_{p}^{2}\right| \psi_{i}>=M$ nucléaire qui intervient dans la désintégration de ce niveau, et, si le transfert d'impulsion n'est pas trop grand, dans l'excitation coulombienne par électrons. Aucun photon transversal ne peut intervenir au premier ordre dans ces processus ; au deuxième ordre, nous n'avons que l'interaction coulombienne entre la densité de charge nucléaire et la densité de charge du champ électronique et l'émission de deux photons. L'élément de matrice $M$ de transition des processus à électrons est proportionnel à

$<\Phi_{f}($ noyau $) \psi_{f}($ électron $)\left|\sum_{1}^{z} \frac{1}{\left|r_{e}-r_{p}\right|}\right|$

$\Phi_{i}($ noyau $) \psi_{i}($ électron $)>$ ce qui, en introduisant la transformée de Fourier à $\frac{1}{2 \pi^{2}} \frac{1}{q^{2}}$ de $\frac{1}{\rho}$ devient

$$
\begin{aligned}
\int \frac{d^{3} q}{q^{2}}<\Phi_{f}(\text { noyau }) & \left|\sum_{1} \mathrm{e}^{i q r_{p}}\right| \Phi_{i}(\text { noyau })> \\
& <\psi_{f} \text { (électron) } \mathrm{e}^{-i q r_{\mathrm{e}}} \psi_{i}(\text { électron })>
\end{aligned}
$$

Avec $J_{i}=0, J_{f}=0$, eiqr peut être remplacé par $j_{\mathrm{c}}\left(q r_{p}\right)$ et si les transferts d'impulsion ne sont pas trop grands par $1-q^{2} r_{p}^{2} / 6$. D'où

$$
\begin{aligned}
& M r_{p}^{2}-<\Phi_{f}(\text { noyau }) \sum_{1}^{*} r^{p} \div \\
& \qquad \Phi_{i}(\text { noyau })><\psi_{f}(0) \mid \psi_{i}(0)>.
\end{aligned}
$$

Tous les processus électroniques ne diffèrent alors que par les fonctions d'onde électroniques, et l'information nucléaire est toujours l'élément de matrice monopolaire. Celui-ci peut être mis sous la forme $M_{\text {rucl. }}=\rho R^{2}, \rho$ étant sans dimensions, et $R$ le rayon du noyau.

Les valeurs expérimentales de $\rho$ connues sont

$\begin{array}{ccccccc}{ }^{12} \mathrm{G} & { }^{16} \mathrm{O} & { }^{40} \mathrm{Ca} & { }^{70} \mathrm{Ge} & { }^{72} \mathrm{Ge} & { }^{90} \mathrm{Zr} & { }^{214} \mathrm{Po} \\ 0,5 & 0,42 & 0,15 & 0,09 & 0,11 & 0,056 & 0,17\end{array}$

Si l'on compare les valeurs expérimentales et les valeurs théoriques calculées sur la base des modèles mentionnés plus haut, la conclusion générale est la suivante : les modèles collectifs conduisent à des valeurs trop élevées de l'élément de matrice monopolaire ; tandis qu'un modèle fondé exclusivement sur une excitation d'une paire de nucléons (Schiff 1955 [9]) à une valeur trop petite, ainsi qu'en témoigne le tableau suivant, pour ${ }^{16} \mathrm{O}$.

Élément de matrice $M$ nucléaire en $10^{-26} \mathrm{~cm}^{2}$

Expérimental Modèle $\alpha$ hydrodynamique Griffin Schiff
3,8
17
19
$17 \quad 0,6$

Les valeurs de Perring et Skyrme [11] et Ferrell et Visscher [9] sont déja plus près de la réalité, parce qu'impliquant un mélange des deux extrêmes. Dans ces conditions il serait peut-être intéressant d'avoir une information supplémentaire, et celle-ci peut être donnée par l'émission de deux photons.

Les éléments de la théorie générale de cette émission ont été donnés par M. Goeppert-Mayer (1931) [4] et Jacob et Eisler (1959 [6]). Adaptée au cas qui nous intéresse, cette théorie donne les résultats suivants :

1. Le spectre d'énergie des deux photons est en $\omega^{3} \omega^{13}$ pour les deux cas $2 E_{1}$ et $2 M_{1} ; 2 E_{2}$ est beaucoup moins probable, même si l'on tient compte du fait que l'on rencontre des états intermédiaires $2^{+}$d'énergie moins élevée.

2. La corrélation angulaire est en $1+\cos ^{2} \theta$, sauf si les transitions $2 E_{1}$ et $2 M_{1}$ sont du même 
ordre, dans quel cas il y a un terme d'interférence en $\cos \theta$.

3. La probabilité totale de transition $W\left(2 E_{1}\right)$ est donnée par

$$
W\left(2 E_{1}\right)-1,310^{6} \Delta^{5} \times D^{2}
$$

où $\Delta$ est l'énergie disponible en $\mathrm{MeV}$,

$$
\left.D=\sum_{g} \frac{\Delta}{E_{i}-E_{g}-\Delta / 2}<f|Z| g\right\rangle\langle g|Z| i>,
$$

$Z$ la composante suivant un axe de l'opérateur dipolaire adéquat, $g$ désigne un état intermédiaire, $i$ et $f$ les états initial et final. L'élément de matrice $<f|Z| g>$ est celui qui intervient dans les réactions photonucléaires, et par conséquent les états $g$ les plus importants se trouvent dans la résonance géante. Dans ce cas, la différence $E_{i}-E_{0}$ est beaucoup plus grande que $\hbar \omega, \hbar \omega^{\prime}$, ce qui permet de remplacer dans le dénominateur $\hbar \omega, \hbar \omega^{\prime}$ par une valeur moyenne $\Delta / 2$. La probabilité de transition $2 M_{1}$ est donnée par :

avec

$$
W\left(2 M_{1}\right)=1,610^{2} \Delta^{5}|M|^{2}
$$

$$
M=\sum_{\boldsymbol{g}}\left\langle f\left|M_{z}\right| g\right\rangle\left\langle g\left|M_{z}\right| i\right\rangle \quad \Delta / E_{\boldsymbol{i}}-E_{\boldsymbol{g}}-\Delta / 2
$$

$M$ étant la composante du moment magnétique, en magnétons de Bohr. On voit que $W\left(2 E_{1}\right)$ et $W\left(2 M_{1}\right)$ varient comme $\Delta^{7}$, et par conséquent on a avantage de choisir, si l'on veut mettre en évidence l'émission de deux photons, des transitions à $\Delta$ élevé, puisque les autres probabilités de désintégration varient comme $Z \Delta^{5}$ (paires d'électrons) et $Z^{4} \Delta^{2}$ (capture). Les formes données pour les éléments de matrice intervenant dans l'émission de deux photons montrent que celle-ci peut apporter des informations nucléaires nouvelles. Dans le cas de $\mathrm{O}^{+}$, l'excitation $1 s \rightarrow 2 s$ ne contribue pas dans le modèle élémentaire à particules indépendantes (état intermédiaire $1 p$ occupé) tandis que les excitations de paires peuvent donner une contribution $\neq 0$, contrairement à ce qui se passe pour les émissions électroniques.

On peut introduire dans le calcul de $D$ l'approximation suivante : en supposant que la résonance géante épuise pratiquement la règle de somme, et que l'on puisse négliger la largeur de cette réso-

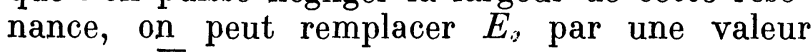
moyenne $\bar{E}_{\boldsymbol{\sigma}}$ et utiliser la relation de fermeture sur les états $g$ pour obtenir

$$
D=\frac{\Delta}{E_{i}-E_{g}-\Delta / 2} \times \frac{1}{3}\left\langle f\left|(R)^{2}\right| i\right\rangle
$$

où

$$
(R)^{2}=\frac{1}{4}\left\{\sum_{i}^{A} r_{i}^{2}+\frac{1}{3} \sum_{i \neq j} r_{i} r_{j} \tau_{z i} \tau_{z j}+\ldots\right.
$$

Si l'on ne prend que le premier terme de $(R)^{2}$, on peut alors se ramener à l'élément de matrice monopolaire, dans quel cas les rapports $W\left(2 E_{2}\right) / W$ (paires + capture) seraient de $2,7.10^{-2}$ et de $0,9 \cdot 10^{-2}$ dans le cas de ${ }^{\mathbf{1 6}} \mathrm{O}$ et de ${ }^{\mathbf{4}} \mathrm{Ca}$. Une déviation significative des résultats expérimentaux par rapport à ces valeurs donne une idée de l'importance de l'excitation de paires de nucléons. Les résultats théoriques pour ${ }^{90} \mathrm{Zr}$ prévoient un rapport $W\left(2 E_{1}\right) / W$ (Paires + capture) de l'ordre de $10^{-4} \grave{a} 10^{-5}$.

En conclusion, on voit que les niveaux $\mathrm{O}^{+}$des noyaux magiques sont loin d'être complètement compris et que l'étude de l'émission de deux photons peut apporter des informations supplémentaires fort utiles pour nous guider dans le choix des configurations susceptibles de décrire ces états.

\section{BIBLIOGRAPHIE}

[1] Bayman (D. F.), Reiner (A. S.), Sheline (R. K.), Phys. Rev., 1959, 115, 1627.

[2] Dennisson (D. M.), Phys. Reo., 1954, 96, 378.

[3] Ferrell (R. A.), Visscher (W. M.), Phys. Res., 1956, 102, 450 .

[4] Goeppert Mayer (M.), Ann. Physik, 1931, 9, 273.

[5] Griffin (J. J.), Phys. Rev., 1957, 108, 311.
[6] JAcob (G.), Eisler (J.), Z. Physik, 1959, 157, 286.

[7] Kurath (D.), Phys. Res., 1956, 101, 216.

[8] Perring (J. K.), Skyrme (T. H. R.), Proc. Phys. Soc., 1955, A 69, 600 .

[9] Schiff (L. I.), Phys. Rev., 1955, 98, 1281.

[10] Talmi (I.), Nucl. Physics, 1960, 19, 225.

[11] Touchard (J.), C. R. Acad. Sc., 1957, 244, 2499. 